\title{
Planetary Protection and Mars Special Regions-A Suggestion for Updating the Definition
}

\author{
Authors: Petra Rettberg, Alexandre M. Anesio, Victor \\ R. Baker, John A. Baross, Sherry L. Cady, \\ Emmanouil Detsis, Christine M. Foreman, Ernst \\ Hauber, Gabriele Gian Ori, David A. Pearce, Nilton \\ O. Renno, Gary Ruvkun, Birgit Sattler, Mark P. \\ Saunders, David H. Smith, Dirk Wagner, \& Frances \\ Westall.
}

Final publication is available from Mary Ann Liebert, Inc., publishers http://dx.doi.org/10.1089/ ast.2016.1472.

Rettberg P, Anesio AM, Baker VR, Baross JA, Cady SL, Detsis E, Foreman CM, Hauber E, Ori GG, Pearce DA, Renno NO, Ruvkun G, Sattler B, Saunders MP, Smith DH, Wagner D, Westall F, "Planetary Protection and Mars Special Regions-A Suggestion for Updating the Definition," Astrobiology, 2016 February; 16(2):119-25. 


\section{Planetary Protection and Mars Special Regions-A Suggestion for Updating the Definition}

Petra Rettberg, Alexandre M. Anesio, Victor R. Baker, John A. Baross, Sherry L. Cady, Emmanouil Detsis, Christine M. Foreman, Ernst Hauber, Gian Gabriele Ori, David A. Pearce, Nilton O. Renno, Gary Ruvkun, Birgit Sattler, Mark P. Saunders, David H. Smith, Dirk Wagner, and Frances Westall

We highlight the role of COSPAR and the scientific community in defining and updating the framework of planetary protection. Specifically, we focus on Mars "Special Regions," areas where strict planetary protection measures have to be applied before a spacecraft can explore them, given the existence of environmental conditions that may be conducive to terrestrial microbial growth. We outline the history of the concept of Special Regions and inform on recent developments regarding the COSPAR policy, namely, the MEPAG SR-SAG2 review and the Academies and ESF joint committee report on Mars Special Regions. We present some new issues that necessitate the update of the current policy and provide suggestions for new definitions of Special Regions. We conclude with the current major scientific questions that remain unanswered regard-ing Mars Special Regions.

Key Words: Planetary protection-Mars Special Regions-COSPAR policy. Astrobiology 16, 119-125.

\section{Introduction}

\subsection{The motivation for planetary protection}

Planetary protection refers to the practice of protect-ing Solar System bodies (i.e., planets, moons, comets, and asteroids) from contamination by terrestrial life (so-called forward contamination prevention) and protecting Earth from contamination by possible life-forms that may be returned with samples from other Solar System bodies (so-called backward contamination prevention). Planetary pro-tection is a guiding principle in the design and operation of interplanetary missions. Planetary protection reflects both the uncertainty in our knowledge of the space environment being explored and the desire of the scientific community to pre-serve the pristine nature of celestial bodies until they can be studied in detail. The planetary protection requirements of future missions may have to be revised based on the results of previous missions. Planetary protection requirements are therefore not static; they must be updated continuously as the results of new missions become available. The concept of planetary protection is enshrined in the 1967 United Nations Treaty on Principles Governing the 
Table 1. Categories of Mission Types as Reported by KMineK and Rummel (2015)

\begin{tabular}{|c|c|}
\hline $\begin{array}{l}\text { Planetary protection } \\
\text { category }\end{array}$ & Solar System target body or mission type \\
\hline Category I & Flyby, orbiter, lander: Undifferentiated, metamorphosed asteroids; Io; others to be defined (TBD) \\
\hline Category II & $\begin{array}{l}\text { Flyby, orbiter, lander. Venus; Moon (with organic inventory); comets; carbonaceous chondrite } \\
\text { asteroids; Jupiter; Saturn; Uranus; Neptune; Ganymede; Callisto; Titan; Triton; Pluto/Charon; } \\
\text { Ceres; Kuiper Belt objects >1/2 the size of Pluto; Kuiper Belt objects }<1 / 2 \text { the size of Pluto; } \\
\text { others TBD }\end{array}$ \\
\hline Category III & Flyby, orbiters: Mars; Europa; Enceladus; others TBD \\
\hline Category IV & Lander missions: Mars; Europa; Enceladus; others TBD \\
\hline \multirow[t]{3}{*}{ Category $\mathrm{V}$} & Any Earth-return mission \\
\hline & "Restricted Earth return": Mars; Europa; others TBD \\
\hline & "Unrestricted Earth return”: Venus; Moon \\
\hline
\end{tabular}

Activities of States in the Exploration and Use of Outer Space, Including the Moon and Other Celestial Bodies, which states that all countries party to the treaty "shall pursue studies of outer space, including the Moon and other celestial bodies, and conduct exploration of them so as to avoid their harmful contamination" (General Assembly of the United Nations, 1967, Article IX).

\subsection{COSPAR role and process}

Internationally, technical aspects of planetary protection are developed through deliberations between space agencies and national and international scientific organizations, and recommendations are made to the Committee on Space Research (COSPAR), an interdisciplinary committee of the International Council of Science, which consults with the United Nations in this area. COSPAR meets regularly to deliberate the merits of the current set of recommendations, and once an intemational consensus is reached, the international planetary policy is updated. The COSPAR Planetary Protection Policy (COSPAR, 2011; Kminek and Rummel, 2015) defines guidelines and specific requirements depending on the mission target and the mission type. These guidelines and specific requirements are based on the actual state of knowledge.

The planetary protection requirements of a specific mission are categorized according to the nature of the target body (e.g., a planet, moon, comet, or asteroid) and the type of encounter the spacecraft will have with it (e.g., flyby, orbiter, or lander). Specific outbound mission target and/or mission type combinations are organized into four planetary protection categories (Categories I to IV), depending on the likelihood that the target body might have gone through chemical evolution that could support or could have supported potential microbial life. Planetary bodies that have little likelihood of having supported microbial life-forms (e.g., Mercury) are assigned to Category I, and no specific planetary protection requirements are levied. However, a mission in which a spacecraft is scheduled to land on a target body that is of interest to studies of origins of life and has a significant chance of contamination by terrestrial life (e.g., Mars) is assigned to Category IV and must undergo stringent cleaning and bioload-reduction procedures. All missions that return extraterrestrial samples to Earth are assigned to Category $\mathrm{V}$, a planetary protection classifi- cation reserved for inbound missions. A detailed categorization of various space exploration targets is shown in Table 1.

New findings about celestial bodies of interest and new knowledge about the limits of life on Earth may require the COSPAR Planetary Protection Policy to be updated. Recommendations by international advisory groups chartered by space agencies, which currently include the National Aeronautics and Space Administration (NASA) and the European Space Agency (ESA), are weighted and assessed in an iterative manner by COSPAR's Panel on Planetary Protection (PPP). Consensus policy recommendations developed by the PPP are then forwarded for discussion and ultimate approval by COSPAR's Bureau and Council prior to becoming official COSPAR policy.

The development of the concept of Special Regions on Mars is a good example of how planetary protection policies evolve as new information becomes available.

\section{Mars Special Regions}

Mars "Special Regions" (SR) define areas where strict planetary protection measures have to be applied before a spacecraft can explore them. The concept of Mars Special Regions was developed as a way to refer to those places where the environmental conditions might be conducive to microbial growth, since so far martian life-forms have not been identified. In particular, this refers to places that might be warm and/or wet enough to support the replication of microbes that might be carried by a terrestrial spacecraft.

The COSPAR Planetary Protection Policy defines Mars Special Regions:

\begin{abstract}
as a region within which terrestrial organisms may be able to replicate, OR a region which is interpreted to have a high potential for the existence of extant Martian life. Given current understanding, Special Regions are defined as areas or volumes within which sufficient water activity AND sufficiently warm temperatures to permit replication of terrestrial organisms may exist. In the absence of specific information, no Special Regions are currently defined on the basis of Martian life.
\end{abstract}

The COSPAR Planetary Protection Policy (currently approved version COSPAR, 2011; described by Kminek and 
Rummel, 2015) for Special Regions defines them as being those regions constrained by the following parameters:

- Lower limit for water activity: 0.5 ; upper limit: 1.0

- Lower limit for temperature: $-25^{\circ} \mathrm{C}$; no upper limit defined

- Timescale within which limits can be identified: 500 years

\subsection{How the current policy was established}

Observations conducted by NASA's Mars Global Surveyor in the late 1990 s and early 2000 s led to the discovery of transient activity in martian gullies suggesting that liquid water may have flowed on the surface of Mars in recent times (see, e.g., Malin and Edgett, 2000). This discovery had an important impact on planetary protection, demonstrating that some regions of Mars may be more suitable for life than others (Meltzer, 2011).

In April 2002, COSPAR and the International Astronomical Union convened a workshop in Williamsburg, Virginia, to discuss planetary protection policies (Rummel et al., 2002). The workshop resulted in a revision of COSPAR's policies and, in particular, established a new mission category - Category IVc - for spacecraft accessing a Mars Special Region (COSPAR, 2003, pp 67-74). In 2005, NASA adopted COSPAR's concept of Special Regions within its planetary protection policy. In addition, NASA requested the National Research Council (NRC) conduct a study to assess the body of policies, requirements, and techniques designed to protect Mars from organisms originating from Earth that could interfere with and compromise scientific investigations (NRC, 2006, p 1).

The resulting NRC report, "Preventing the Forward Contamination of Mars," concluded that there was insufficient data to distinguish between Mars Special Regions and Mars regions that are not special (NRC, 2006). The committee proposed a new classification system, which would replace COSPAR's Categories IVa through IVc, with $\mathrm{Ca}$ tegory IVn for Non-Special Regions and Category IVs for Special Regions (NRC, 2006). In addition, the NRC committee commented: "Until measurements are made that permit distinguishing confidently between regions that are special on Mars and those that are not, NASA should treat all direct-contact missions (i.e., all category IV missions) as Category IVs missions"' (NRC, 2006, pp 118-119). In other words, the NRC recommended that all of Mars be considered a Special Region until additional observational data with better resolution can be obtained. If implemented, this recommendation would require that all Mars landers be subjected to the most stringent-so-called Viking-levelbioload reduction procedures.

The programmatic consequences of subjecting all Mars landers to Viking-level bioload reduction led NASA to request that the Mars Exploration Program Analysis Group (MEPAG) charter a Science Analysis Group (SAG) to look at Special Regions. In particular, the MEPAG group-SRSAG-was asked "to develop a quantitative clarification of the definition of 'special region' that can be used to distinguish between regions that are 'special' and 'nonspecial" "and to undertake "a preliminary analysis of specific environments that should be considered 'special' and "non-special", (Beaty et al., 2006, p 677).
The SR-SAG found that COSPAR's definition of Special Regions needed additional clarification; specifically, use of the words propagate and likely, which can have different meanings and interpretations (Beaty et al., 2006, p 684). The SR-SAG also constrained physical variables that could be used to define a Special Region, such as the following: how long they exist (about 100 years), the maximum depth of penetration by a spacecraft (about $5 \mathrm{~m}$ into the crust), and the lower limit for the replication of terrestrial life in terms of temperature $\left(-15^{\circ} \mathrm{C}\right.$ or $-20^{\circ} \mathrm{C}$ including margin) and water activity ( 0.62 or 0.5 including margin) (Beaty et al., 2006, pp 684-691). The SR-SAG report concluded by proposing a new definition of "Special Region" that retained the original COSPAR definition and added to it a set of clarifications and implementation guidelines (Beaty et al., 2006, p 719).

In 2007, COSPAR held a Mars Special Regions Colloquium, with the goal of reviewing the conclusions and recommendations contained in both the $2006 \mathrm{NRC}$ and MEPAG (Beaty et al., 2006) reports and devising a consolidated definition of Special Regions. The report of the COSPAR colloquium (Kminek et al., 2010) disagrees with the NRC 2006 report by stating that there is sufficient data to distinguish between "special" and "non-special regions," and it differs from the SR-SAG report by reducing the lower temperature limit for the replication of terrestrial life from $-20^{\circ} \mathrm{C}$ to $-25^{\circ} \mathrm{C}$ (Kminek et al., 2010).

The colloquium report also recommended that the definition of a Special Region and the list of terrains classified as "special" be reviewed every 2 years (Kminek et al., 2010). MEPAG created a new Science Analysis Group (SR-SAG2) in the latter part of 2014 to revisit the concept of Special Regions on Mars following the recommendation of the COSPAR colloquium to review the standards every 2 years.

\subsection{Updating the current policy based on new findings}

2.2.1. The MEPAG SR-SAG2 report. The SR-SAG2 used the following general approach (Rummel et al., 2014):

- Clarifying the terms in the existing COSPAR definition;

- Establishing temporal and spatial boundary conditions for the analysis;

- Reviewing the data sets on the limits of microbial life and the availability of water on Mars;

- Identifying applicable threshold conditions for propagation;

- Evaluating the distribution of the identified threshold conditions on Mars;

- Analyzing on a case-by-case basis those purported environments on Mars that could potentially meet or exceed the biological threshold conditions;

- Describing conceptually the possibility for spacecraftinduced conditions that could exceed the threshold levels for propagation; and

- Considering the impact of Special Regions on potential future human missions to Mars.

The resulting SR-SAG2 report provided a comprehensive distillation of the current understanding of the limits of terrestrial life and relevant martian conditions and presented an analytical approach for considering Special Regions using current and future improvements in knowledge. The SR- 
SAG2 report determined that the lower temperature limit should be $-28^{\circ} \mathrm{C}$ (including a margin of $10^{\circ} \mathrm{C}$ ) and the water activity $\left(a_{\mathrm{w}}\right)$ should be above 0.60 (Rummel et al., 2014, pp 894-898). It also updated the list of features on Mars that should be classified as "special," "non-special," and "uncertain" regions ${ }^{1}$

\subsection{The Academies and European Science Foundation review of the MEPAG SR-SAG2 report}

2.3.1. The joint committee for the review of the MEPAG SR-SAG2 report. Two events occurred nearly simultaneously in October 2014, while the SR-SAG2 report was being prepared for publication in the November 2014 issue of the joumal Astrobiology. First, ESA requested that the European Science Foundation (ESF) conduct a review of the SR-SAG2 report. Second, the Associate Administrator of NASA's Science Mission Directorate approached the Space Studies Board (SSB) of the National Academies of Sciences, Engineering, and Medicine with a request "to review the conclusions and recommendations contained in the SR-SAG2 report and assess their consistency with current understanding of both the martian environment and the physical and chemical limits for the survival and propagation of microbial and other life on Earth."

NASA and ESA maintain a close working relationship, as do their respective planetary protection offices. Similarly, the SSB and the ESF's European Space Science Committee maintain a cooperative relationship and have published a number of joint reports over the past several decades. It made no sense for the two organizations to review independently the same document. Therefore, with the concurrence of the ESA and NASA planetary protection officers, the Academies and ESF joined forces and developed the following statement of task for a joint review of the SR-SAG2 report:

An ad hoc committee under the auspices of the Academies and the European Science Foundation review the current planetary protection requirements for Mars Special Regions and their proposed revision as outlined in the 2014 Special Regions report of the Mars Exploration Program Analysis Group (MEPAG). The resulting report from the review shall include recommendations for an update of the planetary protection requirements for Mars Special Regions.

The report of the review committee was published in September, 2015, as a joint report of the Academies and ESF under the title "Review of the MEPAG Report on Mars Special Regions" (free copy of the report in PDF format can be downloaded from http://www.nap.edu/catalog/21816/ review-of-the-mepag-report-on-mars-special-regions) (SSB and ESF, 2015).

\footnotetext{
${ }^{1}$ According to the SR-SAG2 report (p 888), uncertain regions are defined as follows:

Uncertain Regions. If a martian environment can simultaneously demonstrate the temperature and water availability conditions identified in this study, propagation may be possible, and those regions would be identified as Special Regions. Nonetheless, because of the limited nature of the data available for regions only sensed remotely, it may not be possible to prove that such environments are capable of supporting microbial growth. Such areas are therefore treated in the same manner as Special Regions until they are shown to be otherwise.
}

2.3.2. The rationale for the Academies-ESF report. It might, at first sight, appear anomalous to go to the trouble and expense of convening a committee with a membership spanning two continents to review a document that had already been published in a peer-reviewed joumal. But, as noted in the joint report (SSB and ESF, 2015, preface page xii):

The planetary protection policies of both NASA and ESA, in accord with COSPAR policy, entail that planetary protection requirements imposed on spaceflight missions be determined following receipt of multidisciplinary scientific advice. ESF and the Academies provide unique interface with their respective scientific communities through their membership organizations and can provide independent advice taking into account all relevant areas of science, including the engineering and social sciences and the humanities. As a consequence, both NASA and ESA have established arrangements by which the Academies and ESF, respectively, provide strategic advice on planetary protection.

It is worth noting that, in addition to reviewing the MEPAGSAG2 report, the joint review committee provided COSPAR with an update of the results of relevant publications and mission findings since the release of the MEPAG-SAG2 report.

The joint report, together with the MEPAG-SAG2 report, was presented and discussed at an international workshop, organized by COSPAR's PPP, in Bern, Switzerland, on September 22-24, 2015. The workshop and successor activities are part of the COSPAR process used to revise and update planetary protection policies. Recommendations from the PPP will ultimately be forwarded to COSPAR's Bureau and Council for action and potential incorporation in COSPAR planetary protection policy.

The findings from the SR-SAG2 report were discussed by the committee in view of additional information contained in scientific publications not addressed by the SR-SAG2 report and from new knowledge obtained by ongoing space missions, field studies, and laboratory experiments since the publication of the MEPAG SR-SAG2 report. This included discussions about the breadth and depth of SR-SAG2 analysis with respect to survivability of life-forms singularly versus in communities, and the SR-SAG2 approach used to define geographical areas as Special Regions. The review committee agreed with most of SR-SAG2's individual findings, including retaining the current limits for life specified by COSPAR, but arrived at different conclusions in some cases and is of the opinion that a more detailed consideration is necessary. The reader is directed to the published report (SSB and ESF, 2015) for the detailed discussion on each finding. Here, we will report on the two significant new discoveries that drive the update of the definition of Mars Special Regions, which were published after the MEPAG SR-SAG2 report (and thus were not included in the report).

2.3.3. Methane on Mars. Trace concentrations of methane in Mars' atmosphere were measured recently by an in situ spectrometer on the Mars Curiosity Rover (Webster et al., 2015). Trace amounts of methane had been identified earlier by ground-based spectrometers (Krasnopolsky et al., 2004) and orbital spectrometers (Formisano et al., 2004). The Mars atmospheric methane concentration appears to vary seasonally. The source of methane and its variations is unknown and 
can be either of biotic or abiotic origin. As noted by Schuerger et al. (2011), at least eight possible mechanisms may be involved in the production of methane on Mars including outgassing from comet and asteroid impacts, outgassing from interplanetary dust particles, subsurface clathrates, subsurface serpentinization of olivine, UV photolysis of $\mathrm{H}_{2} \mathrm{O}$ in the presence of $\mathrm{CO}$ yielding intermediates that quickly recombine to form methane, geothermal outgassing, presumptive biological processes, and UV catalysis of organics in the martian regolith. A biological origin would make the source of methane on Mars a Special Region. If methane originates from the melting of subsurface clathrates, the processes that would have led to the formation of methane clathrates in the past, in addition to methane production by subsurface serpentinization of olivine, would have involved the presence of liquid water. Therefore, methane can be an indicator of water and temperatures that could support microbial life somewhere in the subsurface of Mars, either now or in the past. The active source and/or reservoirs of methane release on Mars should be considered as a Special Region until proven otherwise.

2.3.4. Recurring slope lineae and slope streaks. Recurring slope lineae (RSL) are narrow ( $<5 \mathrm{~m}$ wide), dark features that occur on steep $\left(25^{\circ}\right.$ to $\left.40^{\circ}\right)$ slopes during warm seasons on low-albedo surfaces (McEwen et al., 2011, 2014; Ojha et al., 2014). The particularity of the RSL is that they grow incrementally, can be more than $1 \mathrm{~km}$ long, and recur over several years. All confirmed RSL locations have warm daily peak temperatures (typically $>273 \mathrm{~K}$ at the surface) during the seasons in which RSL are active (McEwen et al., 2011).

The SR-SAG2 report devoted considerable attention to these surface features. Both committees accepted that, currently, RSL may be caused by an aqueous process and therefore meet the criteria for an Uncertain Region that is to be treated as a Special Region until proven otherwise. Coincidently, 2 days after the publication of the AcademiesESF report, Ojha et al. (2015) reported hydration features in spectra spatially associated with RSL and obtained at peak RSL activity. Hence, RSL may form as a result of contemporary water activity on Mars, specifically brine flows.

Ongoing research further suggests that RSL differ from at least some phenomena classed as "slope streaks" only because of their smaller size and shorter fading time (Mushkin et al. 2014a). For example, Mushkin et al. (2014b) documented observations of some slope streaks with shorter formation and fading timescales. They reported seasonal change and incremental growth of slope streaks near Olympus Mons and Arabia Terra, in direct contrast to the SR-SAG2 report's generalization for the slope streaks as a phenomenon distinct from RSL. Moreover, recent analyses of areas on which slope streaks form suggest that RSL do not have significant inertia, which would make formation via dry granular flow unlikely (Brusnikin et al., 2015). Although Brusnikin et al. (2015) considered slope streaks to be different from RSL (in agreement with the SR-SAG2 report), their results suggest that the formation of slope streaks is far from being understood. These results are sufficient to indicate that more attention needs to be devoted to understanding the relationships between the now intensely studied RSL and at least some of the less well studied features that have been grouped into the general category of "slope streaks." Like RSL, it is advisable that these phenomena be documented on a case-by-case basis for any planned landing ellipse to demonstrate that they really are "dry dust avalanches" and not caused by aqueous processes.

\subsection{Suggestion for an updated definition of Mars Special Regions by the Academies-ESF joint committee}

Given current understanding of terrestrial organisms, Special Regions are defined as areas or volumes within which sufficient water activity and sufficiently warm temperatures to permit replication of Earth organisms may exist. The physical parameters delineating applicable water activity and temperature thresholds are given below:

- Water activity: lower limit, 0.5; upper limit, 1.0

- Temperature: lower limit, $-25^{\circ} \mathrm{C}$; no upper limit defined

- Timescale within which limits can be identified: 500 years

Observed features for which there is a significant (but still unknown) probability of association with liquid water, and which should be considered as Uncertain Regions and treated as Special Regions until proven otherwise:

- Sources of methane;

- Recurring slope lineae;

- Gullies, and bright streaks associated with gullies;

- Pasted-on terrains;

- Caves, subsurface cavities, and subsurface below $5 \mathrm{~m}$; and

- Others, to be determined, including dark slope streaks, possible geothermal sites, fresh craters with hydrothermal activity, or sites of recent seismic activity.

Spacecraft-induced Special Regions are to be evaluated, consistent with these limits and features, on a case-by-case basis.

Organizations proposing to investigate any region that may meet the criteria above have the responsibility to demonstrate, based on the latest scientific evidence and mission approach, whether their proposed landing sites are or are not Special Regions.

In the absence of specific information, no Special Regions are currently identified on the basis of possible martian lifeforms. If and when information becomes available on this subject, Special Regions will be further defined on that basis.

\section{Conclusions}

The internationally accepted aim of planetary protection is the preservation of the pristine nature of celestial bodies to enable the scientific study of chemical evolution and the origins of life in the Solar System, and the protection of Earth from possible life-forms that may be retumed from other Solar System bodies. Updating the COSPAR Planetary Protection Policy is an iterative process involving the scientific community based on new scientific discoveries and/ or a new understanding of scientific observations (Fig. 1).

Obviously, Special Regions are some of the most attractive targets for the search for traces of life. In situ investigations of Special Regions are possible and feasible, taking existing processes and procedures into consideration. The planetary 


\section{Process of updating the COSPAR Planetary Protection Policy}

\section{(1)}
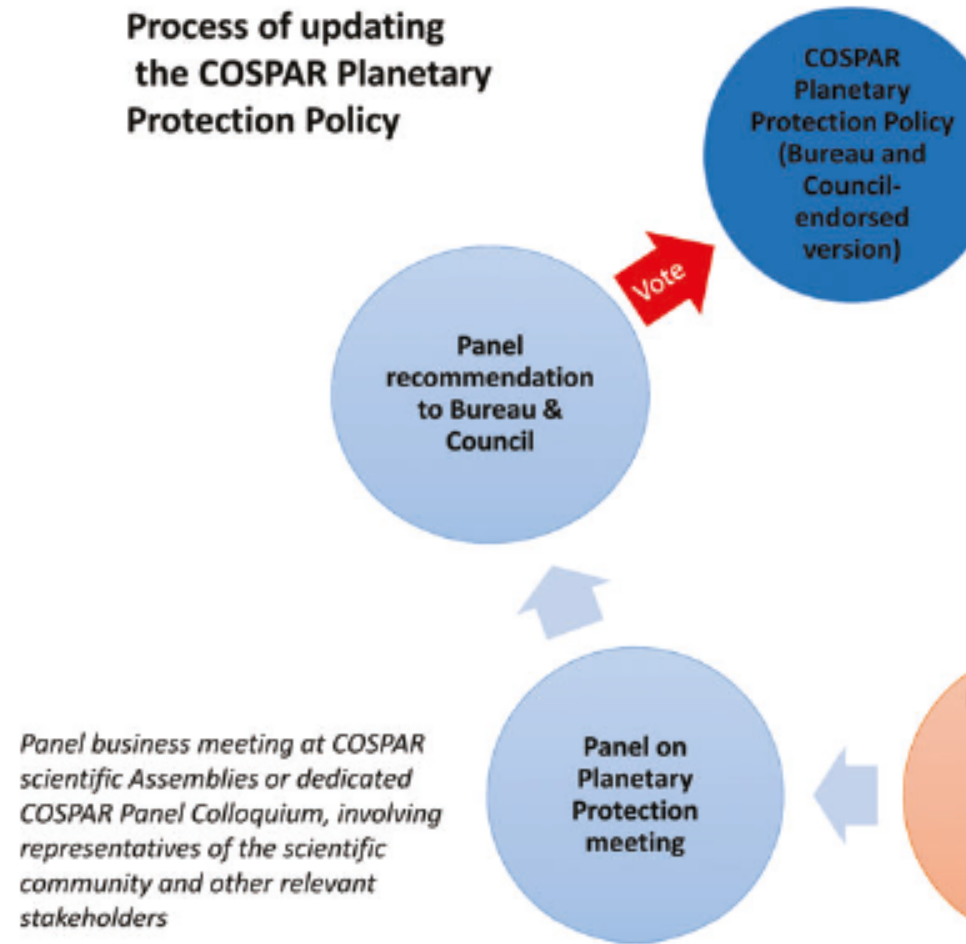

FIG. 1. A schematic of the process of updating COSPAR policy, adapted from Kminek and Rummel (2015).

protection framework exists to ensure that these regions remain free of terrestrial contamination during exploration activities.

\subsection{Critical scientific questions}

During the COSPAR PPP meeting discussion, it became clear that both SR-MEPAG SR-SAG2 and the joint committee have identified a number of critical issues for which answers are not currently known, except perhaps as empirical results in some cases. These issues constitute the "known unknowns," scientific questions that need to be answered in order for the planetary protection community to better refine the concept of Special Regions. These issues are

- Can an organism replicate if it only has access to water vapor and not liquid water? The conditions on Mars do not allow for the presence of pure liquid water. Nevertheless, water vapor could be available to microorganisms. Would that be sufficient for propagation?

- Is replication possible if water activity $\left(a_{\mathrm{w}}\right)$ and temperature $\left(T_{c}\right)$ exceed critical values asynchronously? In several places on Mars it is possible that the water activity and temperature critical values may be exceeded (as defined for Special Regions) but not at the same time. Are there any mechanisms that would allow microorganisms to, for example, store water when the conditions allow it and use it for replication once the temperature reaches the appropriate levels?

- Have experiments to determine lower temperature limit for replication been conducted on sufficiently long timescales to study extremely slow-growing microorganisms? This is a very important questions with regard to the determination of the lowest temperature that cell division is possible.
- Can a single terrestrial organism propagate on Mars even if $a_{\mathrm{w}}$ and $T_{\mathrm{c}}$ are appropriate?

- Do multispecies colonies have an enhanced ability to proliferate in extreme conditions? Are multispecies biofilm a valid survival and proliferation strategy in the martian environment?

- Do physical and chemical conditions in microenvironments mirror those of macroenvironment? The temperature and humidity conditions that define Special Regions are necessarily measured in the macroscale (orbital platforms, rovers, etc). These conditions might not necessarily capture the various microniches available for microorganisms.

\section{Acknowledgments}

This article is based on work supported by the Contract NNH11CD57B between the National Academies of Sciences, Engineering, and Medicine and the National Aeronautics and Space Administration and work supported by the Contract RFP/IPL-PTM/PA/fg/306.2014 between the European Science Foundation and the European Space Agency.

Any opinions, findings, conclusions, or recommendations expressed in this publication are those of the authors and do not necessarily reflect the views of the agency that provided support for the project.

\section{References}

Beaty, D., Buxbaum, K., Meyer, M., Barlow, N., Boynton, W., Clark, B., Deming, J., Doran, P.T., Edgett, K., Hancock, S., Head, J., Hecht, M., Hipkin, V., Kieft, T., Mancinelli, R., McDonald, E., McKay, C., Mellon, M., Newsom, H., Ori, G., 
Paige, D., Schuerger, A.C., Sogin, M., Spry, J.A., Steele, A., Tanaka, K., and Voytek, M. (2006) Findings of the Mars Special Regions Science Analysis Group. Astrobiology 6:677-732.

Brusnikin, E.S., Kreslavsky, M.A., Karachevtseva, I.P., Zubarev, A.E., Patratiy, V.D., and Head, J.W. (2015) Slope streaks on Mars: analysis of geometric parameters [abstract 1115]. In $46^{\text {th }}$ Lunar and Planetary Science Conference, Lunar and Planetary Institute, Houston.

COSPAR. (2003) Planetary Protection Policy, COSPAR Information Bulletin, 156, COSPAR, Paris.

COSPAR. (2011) COSPAR Planetary Protection Policy [20 October 2002, as amended to 24 March 2011], COSPAR, Paris. Available online at https://cosparhq.cnes fr/sites/default/ files/pppolicy.pdf

Formisano, V., Atreya, S.K., Encrenaz, T., Ignatiev, N., and Giuranna, M. (2004) Detection of methane in the atmosphere of Mars. Science 306:1758-1761.

General Assembly of the United Nations. (1967) Treaty on Principles Governing the Activities of States in the Exploration and Use of Outer Space, Including the Moon and Other Celestial Bodies. United Nations, New York. Available online at http://www.unoosa.org/oosa/en/ourwork/spacelaw/ treaties/outerspacetreaty.html

Kminek, G. and Rummel, J. (2015) Research highlights. Space Research Today 193:7-18.

Kminek, G., Rummel, J.D., Cockell, C.S., Atlas, R., Barlow, N., Beaty, D., Boynton, W., Carr, M., Clifford, S., Conley, C.A., Davila, A.F., Debus, A., Doran, P., Hecht, M., Heldmann, J., Helbert, J., Hipkin, V., Horneck, G., Kieft, T.L., Klingelhoefer, G., Meyer, M., Newsom, H., Ori, G.G., Parnell, J., Prieur, D., Raulin, F., Schulze-Makuch, D., Spry, J.A., Stabekis, P.E., Stackebrandt, E., Vago, J., Viso, M., Voytek, M., Wells, L., and Westall, F. (2010) Report of the COSPAR Mars Special Regions Colloquium. Adv Space Res 46:811-829.

Krasnopolsky, V.A., Maillard, J.P., and Owen, T.O. (2004) Detection of methane in the martian atmosphere: evidence for life? Icarus 172:537-547.

Malin, M.C. and Edgett, K.S. (2000) Evidence for recent groundwater seepage and surface runoff on Mars. Science 288:2330-2335.

McEwen, A.S., Ojha, L., Dundas, C.M., Mattson, S.S., Byrne, S., Wray, J.J., Cull, S.C., Murchie, S.L., Thomas, N., and Gulick, V.C. (2011) Seasonal flows on warm martian slopes. Science 333:740-743.

Meltzer, M. (2011) When Biospheres Collide: A History of NASA's Planetary Protection Programs, NASA, Washington, DC.

Mushkin, A., Gillespie, A.R., Montogomery, D.R., Hibbitts, C.A., and Schreiber, B.C. (2014a) A brine extrusion model for recurring slope lineae [abstract 1342]. In Eighth International Conference on Mars, Lunar and Planetary Institute, Houston.

Mushkin, A., Stillman, D.E., Gillespie, A.R., Montgomery, D.R., Schreiber, B.C., and Hibbitts, C.A. (2014b) New constraint on the recurrence, growth and fading characteristics of low-albedo streaks on martian slopes and their possible hydrologic implications. Geological Society of America $A b$ stracts with Programs 46:792.

NRC. (2006) Preventing the Forward Contamination of Mars, The National Academies Press, Washington, DC.
Ojha, L., McEwen, A., Dundas, C., Byrne, S., Mattson, S., Wray, J., Masse, M., and Schaefer, E. (2014) HiRISE observations of recurring slope lineae (RSL) during southem summer on Mars. Icarus 231:365-376.

Ojha, L., Wilhelm, M.B., Murchie, S.L., McEwen, A.S., Wray, J.J., Hanley, J., Massé, M., and Chojnacki, M. (2015) Spectral evidence for hydrated salts in recurring slope lineae on Mars. Nat Geosci 8:829-832.

Rummel, J.D.; COSPAR Planetary Protection Panel. (2002) Report of the Workshop on Planetary Protection Held under the Auspices of the Committee on Space Research (COSPAR) and the Intemational Astronomical Union (IAU) of the International Council for Science (CSU) at Williamsburg, Virginia, USA on 2-4 April 2002, COSPAR, Paris.

Rummel, J.D., Beaty, D.W., Jones, M.A., Bakermans, C., Barlow, N.G., Boston, P.J., and Chevrier, V.F. (2014) A new analysis of Mars "Special Regions": findings of the second MEPAG Special Regions Science Analysis Group (SRSAG2). Astrobiology 14:887-968.

Schuerger, A.C., Clausen, C., and Britt, D. (2011) Methane evolution from UV-irradiated spacecraft materials under simulated martian conditions: implications for the Mars Science Laboratory (MSL) mission. Icarus 213:393-403.

SSB and ESF. (2015) Review of the MEPAG Report on Mars Special Regions, National Academies Press, Washington, DC. Webster, C.R., Mahaffy, P.R., Atreya, S.K., Flesch, G.J., Mischna, M.A., Meslin, P.-Y., Farley, K.A., et al. (2015) Mars atmosphere. Mars methane detection and variability at Gale Crater. Science 347:415-417.

Abbreviations Used
COSPAR $=$ Committee on Space Research
ESA $=$ European Space Agency
ESF $=$ European Science Foundation
MEPAG $=$ Mars Exploration Program Analysis Group
NASA $=$ National Aeronautics and Space
$\quad$ Administration
NRC $=$ National Research Council
PPP $=$ Panel on Planetary Protection
RSL $=$ recurring slope lineae
SAG $=$ Science Analysis Group
SR $=$ Special Regions
SSB $=$ Space Studies Board

